\title{
The Correlation Between Aqueous Humor Flow and IOP Before and After Trabectome: Developing a Grading System to Quantify Flow
}

Toshihiko Ueda · Hirotaka Suzumura · Murray Johnstone •

Shigekazu Uda $\cdot$ Kazuhide Yoshida

Received: January 7, 2018 / Published online: April 10, 2018

(C) The Author(s) 2018

\section{ABSTRACT}

Introduction: To develop a grading system that provides objective quantification of flow through the conventional aqueous humor outflow (AHO) system. The technique gives clinicians an additional assessment option in the evaluation of glaucoma treatment approaches.

Methods: This was a retrospective observational study. This study evaluated the eyes of all primary open-angle glaucoma patients who underwent a Trabectome (NeoMedix Corp., Tustin, CA, USA) procedure with or without cataract surgery in the interval between April and September $2016(n=73)$. The nasal hemisphere was divided into three regions. Utilizing a fourlevel grading system designed for this study, an aqueous humor outflow grade (G0-G3) was

Enhanced digital content To view enhanced content for this article go to https://doi.org/10.6084/m9. figshare.5999225.

T. Ueda $(\bowtie) \cdot S$. Uda

Nihonmatsu Eye Hospital, Tokyo, Japan

e-mail: ueda@nihonmatsu.net

H. Suzumura

Suzumura Eye Clinic, Tokyo, Japan

M. Johnstone

Department of Ophthalmology, University of

Washington, Seattle, WA, USA

K. Yoshida

Yoshida Eye Clinic, Sōka, Saitama Prefecture, Japan assigned to each region using a video taken during examinations. The individual grade levels of the three regions were combined to get a composite AHO score. The correlation between the composite AHO score and intraocular pressure (IOP) was then analyzed. Additionally, the speed of red blood cell (RBC) clusters in the episcleral veins (ESV) was calculated when made possible by the existence of pulsatile flow.

Results: At 3 months following the Trabectome procedure, average IOP decreased from 26 to $15 \mathrm{mmHg}$. Assessment of the relationship between AHO grade and IOP demonstrated that a high composite AHO score was correlated with lowered IOP (Tukey-Kramer method $p<0.05)$. Additionally, it was found that if one of the three regions had an AHO grade of $\geq \mathrm{G} 2$, an IOP of $<20 \mathrm{mmHg}$ could be predicted. (Fischer's exact test $p<0.0001)$. Calculated speed was as follows: at G1, the speed was $0.68 \pm 0.26 \mathrm{~mm} / \mathrm{s}(n=7)$, at $\mathrm{G} 2$, the speed was $1.8 \pm 0.84 \mathrm{~mm} / \mathrm{s}(n=5)$, and at G3, the speed was $6.8 \pm 3.3 \mathrm{~mm} / \mathrm{s}(n=6)$.

Conclusion: There was a significant correlation between an increase in the composite AHO score and a decrease in IOP. Additionally, the speed of RBC clusters as they traveled through the ESVs remained consistent for each of the grades, and the span of the speeds from lower to higher grades represented a significant range. These findings suggest that the grading system is a reliable measure of AHO.

Trial registration identifier: UMIN 000031745. 
Keywords: Aqueous humor flow; Intraocular pressure; Pulsatile flow; Trabectome; Trabecular outflow

\section{INTRODUCTION}

In the 1940s, Ascher [1] and Goldman [2] discovered aqueous veins, the first clear demonstration that aqueous flows. Aqueous veins from the anterior chamber containing aqueous humor (AH) merge with episcleral veins (ESVs) containing blood that arises from the iris and ciliary body. These aqueous humor veins (AHVs) can be observed using a slit lamp at the maximum magnification, enabling the direct observation of the mixing of red blood cells (RBC) and $\mathrm{AH}$ in the ESVs; AHVs are most numerous on the nasal side of the eye (Fig. 1). Currently, there is considerable interest in exploring the relationship between intraocular pressure (IOP) and aqueous humor outflow (AHO). In addition, there is recognition of a need for a method of quantification of the relationship as is illustrated by the recent work

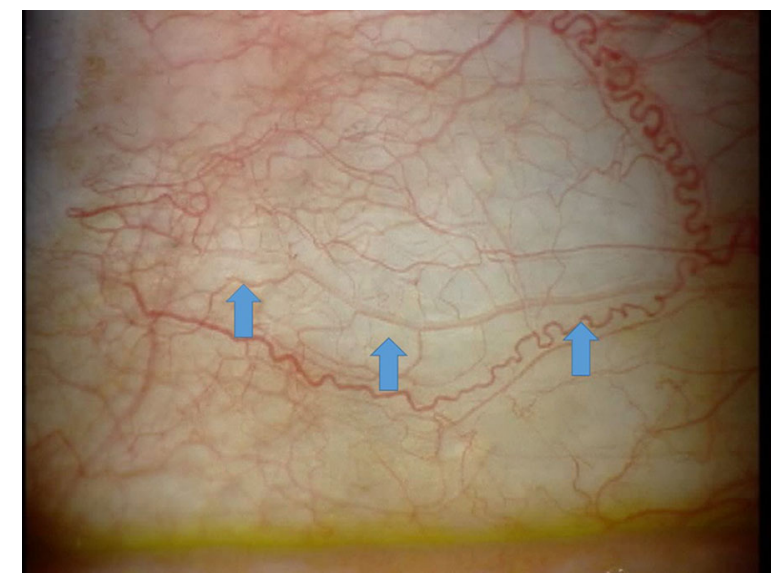

Fig. 1 Aqueous humor outflow (AHO) in the episcleral vein (ESV) of a patient 1 postoperaptive week following a Trabectome procedure. IOP is $14 \mathrm{mmHg}$. The blue arrows point out the flow in the ESVs originating from the limbal region on the left. The proximal section is aqueous-rich. As the red blood cell (RBC) clusters from tributary ESVs merge with the aqueous vein, the stratification of the lamina in the vein can be observed. The winding vessel that appears below is the anterior ciliary artery of Bostan [3] in which the quantitative relationship was investigated after iStent surgery.

In a Trabectome (NeoMedix Corp., Tustin, CA, USA) procedure, the trabecular meshwork (TM) is ablated. If there was no obstruction from the outer wall of Schlemm's canal (SC) to the ESV, it would be logical to assume that the Trabectome procedure would lead to an increase of AHO and a decrease in IOP. In this study, the IOP-lowering effect and the AHO level of the ESVs in the nasal hemisphere were directly observed, measured and compared after Trabectome procedures. The purpose of this study was to develop a grading system to quantify AHO levels and correlate them with IOP changes following Trabectome procedures.

\section{METHODS}

This was a retrospective observational study conducted at Nihonmatsu Eye Hospital in Tokyo, Japan, between April and September 2016. A retrospective chart review was performed for all patients who underwent a Trabectome procedure of approximately $120^{\circ}$ in the nasal hemisphere of the eye with or without phacoemulsification and intraocular lens implant. All surgeries were performed by two surgeons (TU and HY). The patients included in the study were those diagnosed with primary open-angle glaucoma (POAG). Excluded from the study were patients who had an additional diagnosis of pterygium, pinguecula or any condition that might interfere with the observation of AHVs in the nasal hemisphere of the eye. Uveitis or any other conditions that could cause a postoperative elevation of the IOP during the observation period were also excluded. All patients underwent a preoperative gonioscopic examination that revealed an open angle and identifiable landmarks, notably the scleral spur and TM. Combined procedures were performed on patients who also had visually significant cataracts requiring surgical treatment. Written consent was obtained from all patients prior to surgery. All procedures performed in the study involving human participants were in accordance with the ethical standards of the institutional and/or national research 
committee and with the 1964 Helsinki Declaration and its later amendments or comparable ethical standards.

After the surgical procedure, three types of eye drops were prescribed: levofloxacin $(0.5 \%$ Cravit $^{\circledR}$ : Santen Pharmaceutical Co., Ltd., Osaka, Japan), betamethasone sodium phosphate $\left(0.1 \%\right.$ Rinderon ${ }^{\circledR}$ : Shionogi \& Co., Ltd., Osaka, Japan) and pilocarpine hydrochloride (2\% Sanpilo, Santen Pharmaceutical Co., Ltd., Tokyo, Japan), to be administered four times per day for a period of 1 month. All anti-glaucoma drugs were stopped immediately after surgery, except pilocarpine. Clinical information, obtained through chart review, was collected for the following postoperative visits: 1 day, 1 week, 2 weeks, 1 month, 2 months and 3 months. At each follow-up visit, the following data were collected: visual acuity, IOP, any complications and patient gonioscopic findings. IOP was measured using an applanation tonometer.

Digital videos taken during the examinations were used to evaluate and grade the AHO. To eliminate bias in the grading procedure, the IOP and patient details were unknown to the examiner at the time that the AHO grade was assigned. Data were obtained with a slit lamp (Haag Strait BQ 900) at a magnification of 40, which limited the viewing area to $2 \times 2.5 \mathrm{~mm}$. To capture vessels of the entire nasal hemisphere at the high magnification, the hemisphere was divided into three limbal regions, which are referred to using labels that correspond to clock hours. The "upper region," which corresponds to 12:00-2:00 in the right eye and 10:00-12:00 in the left eye, the "middle region," which is identified as 2:00-4:00 in the right eye and 8:00-10:00 in the left eye, and finally the "lower region," which corresponds to 4:00-6:00 in the right eye and 6:00-8:00 in the left eye (Fig. 2). Using Aviutl software, videos of 30 frames per second were taken, allowing a view of the RBC clusters as they travel through the vessels.

RBC clusters result from an oscillatory equilibrium between the aqueous vein and episcleral vein pressures during pulsatile aqueous flow. During diastole, ESV pressure is higher than AHV pressure. An RBC cluster enters the

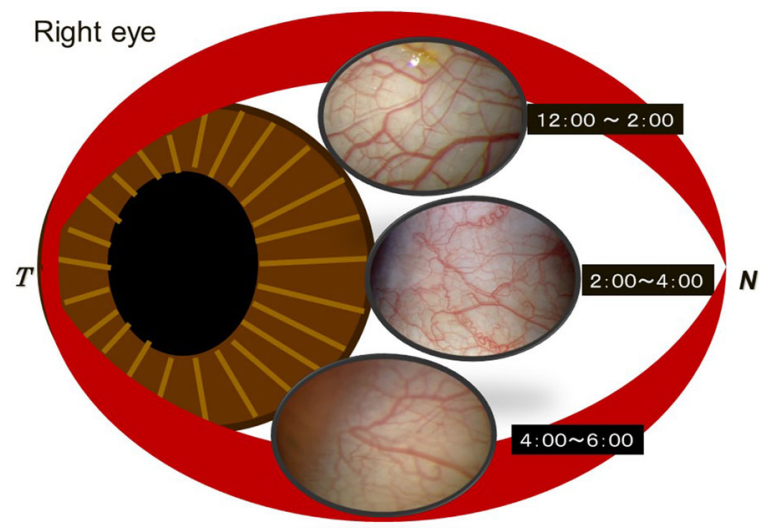

Fig. 2 Aqueous humor outflow (AHO) grade regions. Digital videos taken during the examinations were used to evaluate and grade the AHO. The nasal side was divided into three sections. In the right eye 12:00-2:00 and in the left eye 10:00-12:00 (upper region); 2:00-4:00 in the right eye and 8:00-10:00 in the left eye (middle region); 4:00-6:00 in the right eye and 6:00-8:00 in the left eye (lower region). When using a slit lamp camera at a magnification of 40 , the field of vision is 2 clock hours, making it natural to assign one view to one section

aqueous vein from episcleral vein tributaries. During the next systole, AHV pressure is higher, and the cluster of red cells that entered the aqueous vein in diastole is swept away along the aqueous vein during the systolic aqueous pulse wave.

A piece of graph paper with squares of $1 \mathrm{~mm}$ in size was superimposed onto the video image to confirm and standardize the size. This standardization of size and length enabled the calculation of the speed of RBC clusters as they traveled through the AHVs during pulsatile flow. RBC clusters travel within the moving aqueous humor. Measurement of the time it takes RBC clusters to travel an established distance along an AHV permits determination of the speed of aqueous humor motion. Assuming a vein diameter of approximately $100 \mu \mathrm{m}$, it is further possible to calculate the volume of $\mathrm{AH}$ exiting the eye from an individual AHV.

A 30-s clip was used for each examination; the first $10 \mathrm{~s}$ were generally disregarded to eliminate speed changes caused by fluctuations in IOP after the patient had moved or blinked. For the purpose of this study, evaluation was done on veins that were $\geq 50 \mu \mathrm{m}$ in diameter. 
One aqueous vein was observed in each of the three regions. If more than one vein was visible, the vein with the higher score was used. If there was no visible vein, as sometimes occurs in the upper region, a score of G0 was given.

An AHO level in each of the three regions was assigned to one of four categories on an ordinal scale based on the following criteria: The category termed G0 was used to describe a situation in which the uniform color of the vein made it impossible to observe flow (Fig. 3). The presentation of a vein in this category would appear a dark red. G1 indicated a mixed flow that consisted of more RBC than AH. Veins in this category appear pale red with prominent alternating bands of RBC and $\mathrm{AH}$ (Fig. 4). The slow speed of the RBC permits counting. G2 indicated a level of flow mid-way between G1 and G3, characterized by a pink color with prevalent clusters of RBC that are moving too quickly to be counted (Fig. 5). Finally, G3 indicated a situation in which $\mathrm{AH}$ was predominantly present in the vein. RBCs are present, but they are moving at a speed at which individual clusters cannot be identified, giving an overall impression of white (Fig. 6; Table 1). The assigned scores of G0-G3 for each of the three

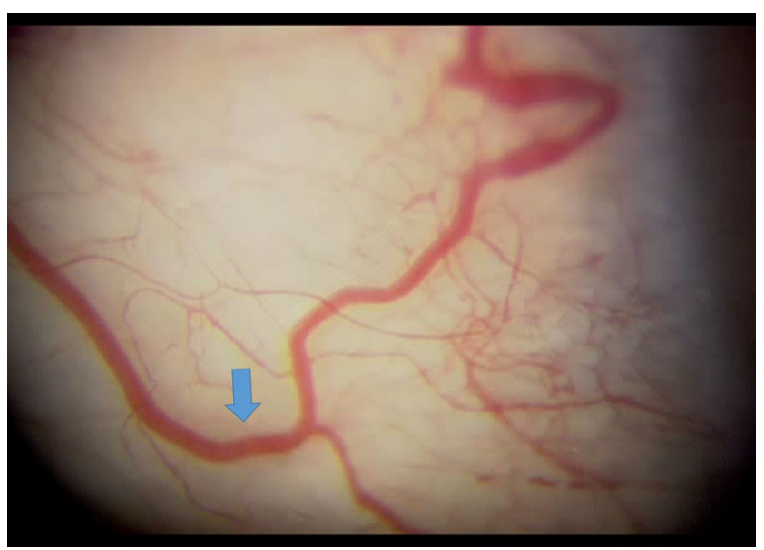

Fig. 3 Aqueous humor outflow (AHO) grade 0. The following three figures demonstrate the clinical presentations of each of the grades. Veins are evaluated in terms of both the color and speed of red blood cell (RBC) clusters as they travel through the vein. Grade 0 is characterized by veins in which the movement of RBC clusters cannot be recognized because of the uniform, deep red vein color. The blue arrows indicate an episcleral vein assigned a score of G0

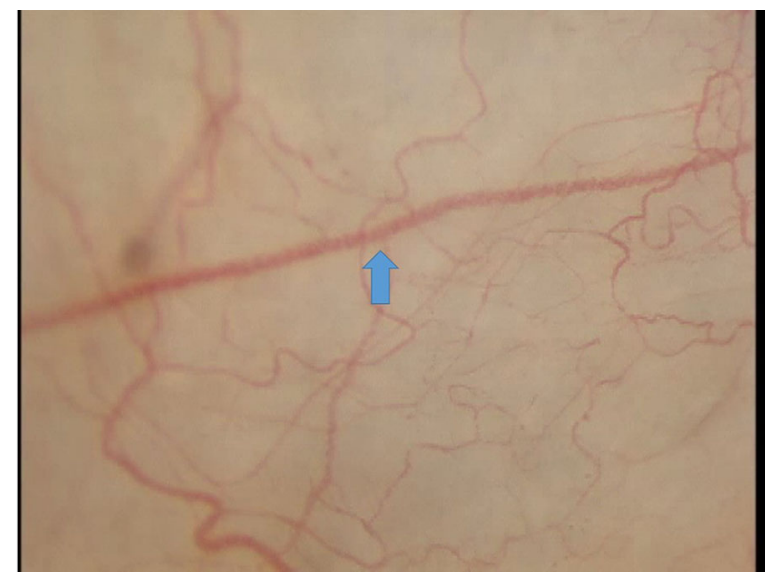

Fig. 4 Aqueous humor outflow (AHO) grade 1. Red blood cell (RBC) clusters can be seen and counted. The color presentation of veins in this category is a pale red, and bands of red clusters mixed with the aqueous humor $(\mathrm{AH})$ can be seen. The blue arrows indicate episcleral veins assigned a score of G1

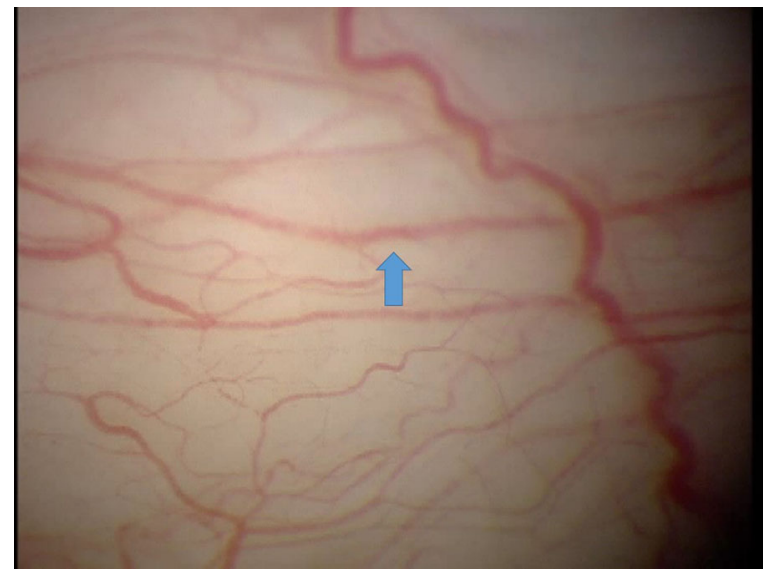

Fig. 5 Aqueous humor outflow (AHO) grade 2. Red blood cell (RBC) clusters are identifiable, but are moving through the vein so quickly that they cannot be counted. Veins in this category are pink in color with noticeable clusters of RBCs. The blue arrows indicate episcleral veins assigned a score of G2

regions were then added together to get a composite AHO score ranging from 0 to 9 .

\section{STATISTICAL ANALYSIS}

Dunnett's test was used to calculate the statistical significance between the preoperative IOP 


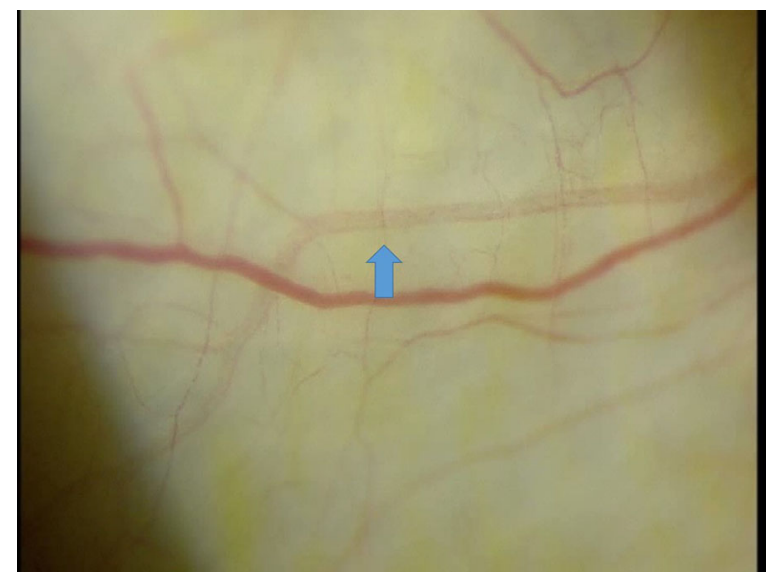

Fig. 6 Aqueous humor outflow (AHO) grade 3. Red blood cells (RBCs) are present, but are moving so fast that individual clusters cannot be identified. The overall impression of the color of veins in this category is white. The blue arrows indicate episcleral veins assigned a score of G3

compared with the postoperative IOP at each of the visits. The statistical significance of the composite AHO grade score compared with the IOP was analyzed using the Tukey-Kramer method, a method of statistical analysis that compares all possible pairs of the means in what could be termed a round-robin fashion. Fisher's exact test is used to test the statistical significance between two nominal variables; in this case, an IOP of $>$ or $<20 \mathrm{mmHg}$ compared to an
AHO level of between G0 and G1 or between G2 and G3, respectively, in the individual regions.

\section{RESULTS}

Two cases were excluded because of a postoperative diagnosis of uveitis not related to the procedure. In these two cases, an unexpectedly high IOP was noted, and an examination showed keratic precipitates, cells and flares in the anterior chamber, leading to a preliminary diagnosis of herpes uveitis. The patients responded to topical steroids and topical antiviral agents. The results of these two cases were excluded and treated as outliers to avoid skewing the overall results. Data from a total of 63 cases involving 71 eyes were included in this study. The average age was $72.58 \pm 9.67$ years old, and the average preoperative eye drop usage was $3.51 \pm 0.97$ medications.

At the preoperative examination, the average IOP $(n=71)$ was $25.56 \pm 8.05 \mathrm{mmHg}$ (Table 2). One day postoperatively, the IOP was $17.8 \pm 7.6 \mathrm{mmHg}(n=71)$, a sudden and significant decrease with a wide standard deviation range. During the subsequent 3 months, however, the average IOP gradually decreased further to $15.2 \pm 2.2 \mathrm{mmHg}(n=71)$, while at the same time there was a considerable reduction in the SD range. There was a statistically significant difference between the preoperative IOP

Table 1 Criteria used to assign the AHO grade level

\begin{tabular}{|c|c|c|}
\hline Grade & RBC speed & Color \\
\hline 0 & Movement cannot be recognized & Deep red \\
\hline 1 & $\begin{array}{l}\text { RBCs can be observed and are moving slowly enough to be } \\
\text { counted }\end{array}$ & $\begin{array}{l}\text { Pale red, with predominant bands of red clusters } \\
\text { with aqueous humor also observable }\end{array}$ \\
\hline 2 & $\begin{array}{l}\text { RBCs move through the vein in clusters at a speed at which } \\
\text { they cannot be counted }\end{array}$ & Pink color with prevalent clusters of RBCs \\
\hline 3 & $\begin{array}{l}\text { RBCs are present, but moving at a speed at which individual } \\
\text { units or clusters cannot be identified }\end{array}$ & White impression \\
\hline
\end{tabular}

Table 1 defines the criteria used to assign grade scores to aqueous veins. Figure 2 shows a visual depiction of the grades. Although the above guidelines provide a general framework to determine the aqueous humor outflow (AHO) grade, ultimately, how to assess and prioritize each of the criteria to form a composite value are up to the clinician's judgment, based on experience. As a general rule, when making an evaluation of the grade, the order of priority was the diameter of the vein, speed of the flow, and color of the vein. $R B C$ red blood cell 
Table 2 Patient characteristics

\begin{tabular}{ll}
\hline Cases & 63 cases, 71 eyes \\
Age & $72.58 \pm 9.67$ \\
Preoperative IOP & $25.56 \pm 8.05$ \\
\hline
\end{tabular}

$I O P$ intraocular pressure, mean \pm standard deviation

and every postoperative IOP (Dunnet's test $p<0.001$ ) (Fig. 7). No patients in this cohort required further surgery or anti-glaucoma eye drops (except for pilocarpine) at 3 months postoperatively.

Figure 8 shows all the IOP values and the composite AHO score of the three regions. All of the values are included here without regard to the time period at which they were taken. The relationship between the IOP (in $\mathrm{mmHg}$ ) and composite AHO score was as follows: score 0 $(n=12)$ had an average IOP of $29.7 \pm 10.6$; score $1 \quad(n=21)$ had an average IOP of $25.3 \pm 9.0$; score $2(n=30)$ had an average IOP of $23.8 \pm 6.7$; score $3(n=70)$ had an average IOP of $20.6 \pm 6.2$; score $4(n=67)$ had an average IOP of $16.8 \pm 4.2$; score $5(n=101)$ had an average IOP of $15.5 \pm 3.6$; score $6(n=82)$ had an average IOP of $14.3 \pm 2.7$; score 7

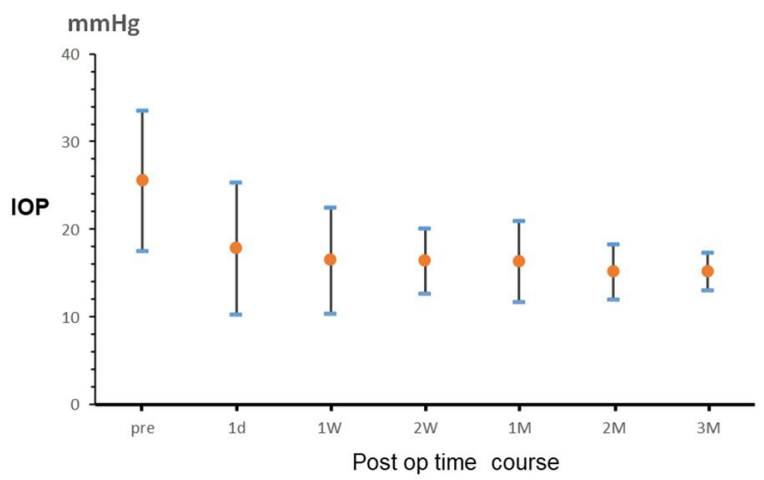

Fig. 7 IOP time course. This graph shows the results over time. The red dot indicates the mean. The blue lines above and below show 1 SD above and below the mean, respectively. Preoperatively, the average IOP was $26 \mathrm{mmHg}$. At 1 day postoperatively, the IOP had significantly decreased, and after 3 months, the average IOP was $15 \mathrm{mmHg}$. There was a statistically significant difference between the preoperative IOP and every postoperative IOP (Dunnet's test $p<0.001$ )

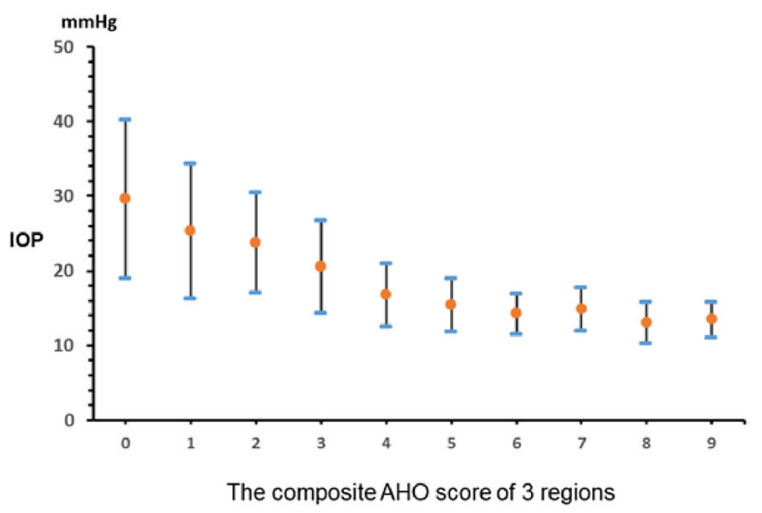

Fig. 8 Correlation between the IOP and composite aqueous humor outflow (AHO) grade level score. Graph interpretation is as specified in Fig. 7. This graph shows the correlation between IOP and the composite score derived from the grading system developed for this study. The scale is from G0, indicating an absence of observable flow, to G9, indicating the maximum flow level. Using the TukeyKramer approach, a method of statistical analysis that compares all possible pairs of means in a round robin fashion, a significant correlation can be seen between grades G0 and G4 ( $p<0.001)$. After G4, however, there is not a significant correlation between higher grade levels and lower IOP

$(n=53)$ had an average IOP of $14.9 \pm 2.9$; score $8(n=15)$ had an average IOP of $13.1 \pm 2.8$; score $9(n=4)$ had an average IOP of $13.5 \pm 2.4$. There is a tendency that as the AHO increases, the IOP decreases. Statistical analysis using the Tukey-Kramer method showed a significant relationship between higher composite AHO scores and lower IOPs for composite scores between 0 and 4 . For composite scores above 4 , however, there was not a significant relationship between higher composite AHO scores and lower IOPs.

By observing the intervals that sometimes occur between RBC clusters as they travel through the ESVs, we can identify the phenomenon of pulsatile flow described by Johnstone [4]. Pulsatile flow produces bands of RBC clusters alternating with $\mathrm{AH}$, allowing us to calculate the speed of AH outflow. Following the frame rate as the clusters travel through the vein, we can calculate the $\mathrm{AH}$ speed in an individual AHV. The average AH speed at G1 was $0.68 \mathrm{~mm} / \mathrm{s}$, at $\mathrm{G} 2,1.8 \mathrm{~mm} / \mathrm{s}$ and at $\mathrm{G} 3$, $6.8 \mathrm{~mm} / \mathrm{s}$ (Table 3). 


\section{DISCUSSION}

The purpose of our study was to develop a grading system to quantify AHO levels and correlate them with IOP changes following Trabectome procedures. Our results indicate that the grading system we developed is able to achieve our objective. We feel that the approach can provide a clinically useful metric for assessing the results of Trabectome and other MIGS procedures because it directly assesses aqueous outflow responses.

There is a large body of evidence to suggest that there is a relationship between IOP and $\mathrm{AHO}$ in the ESVs. It has been noted that diurnal fluctuations in IOP are preceded by fluctuations in AHO [5-8]. Specifically, diurnal decreases in IOP are preceded by increased AHO levels, and diurnal increases in IOP are preceded by a decreased AHO level. Though the temporal relationship makes it natural to assume that increased flow is causing the decreased IOP, and conversely that decreased flow brings about increased IOP, no such causal relationship has been established to date.

Transient rises in IOP associated with increases in $\mathrm{AHO}$ have been induced by many researchers using such techniques as pressure on the side of the eye $[7,9]$, tonometry $[7,9]$ and ophthalmodynamometry $[10,11]$. The rise

Table 3 Speed of the RBC clusters

\begin{tabular}{llll}
\hline Grade & $\boldsymbol{n}$ & $\mathbf{m m} / \mathbf{s}$ & $\mathbf{S D}$ \\
\hline 1 & 7 & $0.68(0.5-1.0)$ & 0.26 \\
2 & 5 & $1.8(1.0-3.0)$ & 0.84 \\
3 & 6 & $6.8(3.0-10.0)$ & 3.3 \\
\hline
\end{tabular}

Using videos taken during the examinations, the speed of aqueous humor $(\mathrm{AH})$ flow was calculated for patients whose aqueous veins (AV) contained both red blood cells (RBCs) and $\mathrm{AH}$. The layered presentation of these mixing veins provides a marker that can be followed along the vein length, permitting calculation of the speed of $\mathrm{AH}$ flow as it travels distally in the outflow system. With a known standard length of $2.5 \mathrm{~mm}$, the speed of the RBC clusters could be calculated. This chart compares the speeds at the different flow levels

$S D$ standard deviation in pressure produced through such techniques is followed by an increase in AHO, which in turn results in an IOP that is lower than the baseline IOP. Flow into aqueous veins stops until the IOP returns to the baseline $[11,12]$.

From these studies, ophthalmologists have come to the conclusion that IOP and AHO have a cause-and-effect relationship. To further explore the proposed relationships, this study examines the degree to which the relationship between IOP and AHO is present after the Trabectome procedure for POAG. Grishaber [13] and Fellman [14] were the first to report clinical observations of AHO in the ESVs using techniques that increase the intraoperative pressure load. In this study, however, AHO was directly observed using a slit lamp, the same technique used by Ascher [1] and Goldman [2]. Johnstone has noted that direct observation of AHO is the most reliable way to judge the flow properties of the outflow system [15]. Ascher [16], referring to aqueous veins, observed, "With high power magnification they can be seen in almost every eye."

The reported occurrence of visible aqueous veins in patients was reported to be $\sim 75 \%$ by Goldmann [9]. In the present study, however, we found that AHO could be directly observed in all cases. An unequal distribution of ESVs in different regions has been shown. The nasal region has been found to contain significantly more ESVs than the temporal region [17]. There is some debate about whether or not the CC ostia are concentrated in the lower nasal region. According to Cha, CCs are concentrated in the lower nasal region [17]. However, Hann [18] reported that CCs are uniformly distributed along the outer wall of SCs. The aqueous outflow may be redistributed to the ESVs by the intrascleral plexus. Therefore, in this study the aqueous outflows of ESVs in the three regions were treated equally. De Vries found that four to five aqueous veins are visible in some eyes, while there may be a maximum of six. However, two to three aqueous veins are typically visible in one eye [10]. Therefore, a composite grade score was calculated by adding the three individual nasal hemisphere scores together to account for the differences in the location of 
preferential flow sites as well as the fact that there may be AHVs elsewhere in the eye.

The grading system developed for this study attempts to identify a way to quantify AHO through direct observation and by assigning a grading score on a scale from G0 to G3 to each of the three regions of the nasal hemisphere. Measuring the IOP has been the standard way to evaluate AHO, but as one author (T.U.) observed in his practice, it is not always true that patients with a high IOP have poor AHO. Therefore, it appears useful to develop a more nuanced picture of the overall condition of the patient's outflow system.

In this cohort (71 cases) all showed good responses, without complications or the need for further interventions during the 3-month follow-up interval. In the early period after a Trabectome procedure, it is not unexpected to see some cases in which the IOP is higher than average. For example, for up to 1 week after the procedure, there might be coagula, debris fragments, viscoelastic material and inflammatory cells in the aqueous humor outflow tract distal to the Schlemm's canal outer wall. Blockage created by these materials may result in a lack of an IOP decrease immediately after a Trabectome procedure. As these blockages naturally resolve over the postoperative time interval, we can see a corresponding decrease in IOP. By 3 months, patients had an average IOP of $15 \mathrm{mmHg}$ with a much narrower range in SD compared with earlier time periods. Figure 7 shows that the 71 eyes included in this study followed the typical recovery course for a successful Trabectome procedure, the ideal patient cohort for the purpose of this study.

There was a significant correlation between the AHO composite grade score and IOP. Additionally, the findings of this study suggest that the speed of $\mathrm{AHO}$ within the different grades remains consistent and increases significantly from $0.68 \mathrm{~mm} / \mathrm{s}$ at $\mathrm{G} 1$ to $6.8 \mathrm{~mm} / \mathrm{s}$ at $\mathrm{G} 3$. The AHO speed again suggests that the grading system developed for this study is indeed measuring discrete categories that provide valuable insight into the physiologic conditions of the patients. Both of these findings suggest that there is validity to the grading system developed for this study.
Assuming a vessel diameter of $100 \mu \mathrm{m}$, we can calculate that a vessel with an AHO level $\geq \mathrm{G} 2$ has the capacity to carry $0.85 \mu \mathrm{l} / \mathrm{min}$ of $\mathrm{AH}$ out of the eye; the amount discharged from a single vessel can account for just under half of the total of $2 \mu \mathrm{l} / \mathrm{min}$ of aqueous humor that is produced and discharged from a typical eye. With each eye containing approximately 2-3 functioning aqueous veins (with up to 6) [10], if one vein is able to carry $0.85 \mu \mathrm{l} / \mathrm{min}$, then it is easy to imagine that the other $2-5$ vessels will be able to collectively discharge the remaining $1.15 \mu \mathrm{l} / \mathrm{min}$ of $\mathrm{AH}$ without difficulty. Our outflow considerations are consistent with the findings of Stepanik [19] who found that flow through a single AHV averages $1.08 \mu \mathrm{l} / \mathrm{min}$.

As discussed previously, the relationship between a lowered IOP and increased AHO level stops being significant at a composite $\mathrm{AHO}$ score $\geq 4$. This can be explained using the above findings. That is to say, a composite score $\geq 4$ means that at least one region has a score of $\geq \mathrm{G} 2$ and therefore has the capacity to handle a large portion of the AHO. Composite $\mathrm{AHO}$ scores $\geq 4$ do not show an increasing impact on the IOP because a grade of $\geq$ G2 in one region is usually sufficient to guarantee adequate outflow (Table 4).

IOP is currently the standard measurement used to both diagnose and assess the efficacy of glaucoma treatment. Rather than relying on IOP only, however, using AHO grade levels as both a reference and substantiation of treatment success can give us a more complete picture of the overall condition of our patients. Moreover, because the grades can be assigned in

Table 4 Maximum flow grade and IOP

\begin{tabular}{lll}
\hline & $<\mathbf{2 0} \mathbf{~ m m H g}$ & $\geqq \mathbf{2 0} \mathbf{~ m m H g}$ \\
\hline$\leqq$ Grade 1 & 19 & 29 \\
$\geqq$ Grade 2 & 290 & 22 \\
\hline
\end{tabular}

Relationship between maximum flow grade and intraocular pressure (IOP), excluding the preoperative evaluation. Maximum flow was selected in one region for each patient. If at least one region has a composite grade score of $\geq \mathrm{G} 2$, an IOP of $<20 \mathrm{mmHg}$ can be reasonably anticipated Fisher's exact probability test $p<0.0001$ 
the course of our daily clinical practice, on living patients, they can provide us with a method to directly observe and quantify AHO without having to perform additional procedures or use specialized equipment.

It is important to note several limitations of this study. One such limitation is that errors in judgment of the AHO grade flow score can occur. For example, in some cases an AV with an AHO grade flow of G3 cannot be recognized because of the transparent presentation of the vein. When only $\mathrm{AH}$ is present, the $\mathrm{AHO}$ is not observable by slit lamp examination. The inability to identify such veins can lead to an AHO grade score lower than what is actually present. Despite this limitation, it should be noted that in the present study, G3 veins were identified in $16 \%$ of the total observations made. When devising this grading scale, careful thought was given to validity and reliability, with importance placed on balancing the objective of making a nuanced assessment of $\mathrm{AHO}$, with the need to be consistent. In this scale, G0 and G3 have easily identifiable color presentations, which make them relatively easy to assess. Levels G1 and G2 are a little more difficult to judge based on the color presentation alone. The way to differentiate these categories is whether or not the RBCs traveling in the vein are countable (G1) or not (G2). Using the two guidelines of speed and color, we believe that clinicians can make reasonably reliable and valid judgments regarding grade levels. Technologies such as the injection of a fluorescein tracer or OCT would otherwise be required to identify and study such veins. Additionally, patients tensing up during the procedure can also lead to misinterpreting the AHO grade level. When a patient is overly tense or holding their breath, the intrathoracic pressure increases and venous return decreases. In this case, the vessels will turn red and can lead to assigning an artificially low AHO grade score.

Another limitation of this grading system is that the values between the scores are not equidistant and therefore must be considered categorical rather than continuous values. Though strictly speaking it is not possible in such a case to add the scores together, in this study, it proved useful to combine the $\mathrm{AHO}$ grade scores for the three regions to obtain a single composite AHO score of between 0 and 9 . Combining the scores for each of the three regions for all patients will control for the variable differences in concentrated high-flow vessels among patients. For this reason, the decision was made to combine all three regions into a composite score for analysis. From the strong correlation between the IOP and composite AHO score, it can be assumed that the grade levels represent tangible and distinct categories; the correlations also suggest that it is valid to think of them when grouped together as representing a continuum of outflow volume. Additionally, the finding that a flow grade $\geq$ G2 in at least one region is strongly correlated with an IOP of $<20 \mathrm{mmHg}$ means that in our clinical practice it is not always necessary to combine the scores of the three regions to provide confirmatory evidence of a lowered IOP.

Another limitation is the brief duration of the study. One of the benefits of the procedures we describe is that they represent a tool that will make it possible to continue to follow this cohort of patients over time to determine whether initially identified relationships remain stable or change if continued postoperative remodeling of the outflow system pathways occurs. For the purposes of the present study, however, we feel that the time period was adequate to establish the basic relationship between IOP and AHO. In addition, while we feel the currently described scoring system is useful, we recognize that the sensitivity of the scoring system is limited by its subjective nature.

A further limitation of the study is that we did not specifically correlate gonioscopic observations with external markings to determine that we had fully accounted for $180^{\circ}$ of Trabectome treatment.

Additionally, though it may be useful to stratify data in a way that would separate the efficacy of phacoemulsification from that of the Trabecutome procedure, we think the relationship between the IOP decrease and AHO grade level is unlikely to change. 


\section{CONCLUSION}

This study developed a grading system to quantify AHO levels and correlate them with IOP changes following Trabectome procedures. The study demonstrates that as IOP decreases, AHO increases. The results show that the IOP-AHO correlation disappears when the AHO composite score is $\geq 4$ and that we can anticipate an IOP $<20 \mathrm{mmHg}$ in such cases. The direct observation of $\mathrm{AHO}$ is a technique available to all clinicians. We conclude that such observations provide valuable information related to the efficacy of Trabectome procedures that can be used in conjunction with IOP data to assess the efficacy of such procedures over time.

\section{ACKNOWLEDGEMENTS}

Funding. No funding or sponsorship was received for this study or the publication of this article. The article processing charges were funded by the authors.

Authorship. All named authors meet the International Committee of Medical Journal Editors (ICMJE) criteria for authorship for this article, take responsibility for the integrity of the work as a whole, and have given their approval for this version to be published.

Additional Editorial Assistance. Writing assistance for the preparation of this manuscript was provided through private arrangement by Kristie Kainuma.

Disclosures. Toshihiko Ueda, Hirotaka Suzumura, Murray Johnstone, Shigekazu Uda and Kazuhide Yoshida have nothing to disclose.

Compliance and Ethics Guidelines. All procedures performed in this study involving human participants were in accordance with the ethical standards of the institutional and/or national research committee and with the 1964 Helsinki Declaration and its later amendments or comparable ethical standards. Written consent was obtained from all patients prior to surgery.
Data Availability. The data sets during and/ or analyzed during the current study are available from the corresponding author on reasonable request.

Open Access. This article is distributed under the terms of the Creative Commons Attribution-NonCommercial 4.0 International License (http://creativecommons.org/licenses/ by-nc/4.0/), which permits any noncommercial use, distribution, and reproduction in any medium, provided you give appropriate credit to the original author(s) and the source, provide a link to the Creative Commons license, and indicate if changes were made.

\section{REFERENCES}

1. Ascher KW. Physiologic importance of the visible elimination of intraocular fluid. Am J Ophthalmol. 1942;25:1174-209.

2. Goldman H. Abfluss des Kammerwassers beim Menschen. Ophthalmologica. 1946;111:146-52.

3. Bostan C, Harasymowycz P. Episcleral venous outflow: a potential outcome marker for iStent surgery. J Glaucoma. 2017;26(12):1114-9.

4. Johnstone M, Martin E, Jamil A. Pulsatile flow into the aqueous veins: manifestations in normal and glaucomatous eyes. Exp Eye Res. 2011;92(5):318-27.

5. Ascher KW. Backflow phenomenon in aqueous veins of normal and of glaucomatous eyes. Am J Ophthalmol. 1944;27:1074-89.

6. Thomassen TL. On aqueous veins. Acta Ophthalmol. 1947;25:369-78.

7. Thomassen TL, Perkins ES, Dobree JH. Aqueous veins in glaucomatous eyes. $\mathrm{Br} \mathrm{J}$ Ophthalmol. 1950;34:221-7.

8. Stepanik J. Diurnal tomographic variations and their relation to visible aqueous outflow. Am J Ophthalmol. 1954;38:629-45.

9. Goldman H. Weitere Mitteilung über den Abfluss des Kammerwassers beim Menschen. Ophthalmologica. 1946;112:344-6.

10. De Vries S. Zichtbare Afvoer Van Het Kemerwater. Drukkerij Kinsbergen, Amsterdam, 1947. 
11. Kleinert $\mathrm{H}$. The compensation maximum: a new glaucoma sign in aqueous veins. Arch Ophthalmol. 1951;46:518-24.

12. Thomassen TL. The venous tension of eyes suffering from simple glaucoma. Acta Ophthalmol. 1947;25:221.

13. Grieshaber MC, Pienaar A, Oliver J, Stegmann R. Clinical evaluation of the aqueous outflow system in primary open-angle glaucoma for canaloplasty. Invest Ophthalmol Vis Sci. 2010;51:1498-504.

14. Fellman RL, Feuer WJ, Grover DS. Episcleral venous fluid wave correlates with trabectome outcomes. Ophthalmology. 2015;122:2385-91.

15. Johnstone M, Jamil A, Martin E. Aqueous veins and open angle glaucoma. In: Schacknow PN, Samples JR, editors. The glaucoma book: a practical, evidence-based approach to patient care. New York: Springer; 2010. p. 65-78.

16. Ascher KW. The aqueous veins: biomicroscopic study of aqueous humor elimination. Springfield: Charles C Thomas; 1961.

17. Cha ED, $\mathrm{Xu} \mathrm{J}$, Gong L, Gong H. Variations in active outflow along the trabecular outflow pathway. Exp Eye Res. 2016;146:354-60.

18. Hann C, Bentley M, Vercnocke A, Ritman E, Fautsch M. Imaging the human aqueous humor outflow pathway in human eyes by three dimensional micro-computed tomogramphy (3D microCT). Exp Eye Res. 2011;92(2):104-11.

19. Stepanik J. Measuring velocity of flow in aqueous veins. Am J Ophthalmol. 1954;37:918-22. 